19 Revue d'histoire du XIXe siècle

Société d'histoire de la révolution de 1848 et des

révolutions du XIXe siècle

$28 \mid 2004$

Religion, politique et culture au XIXe siècle

\title{
Spiritisme et politique
}

\section{Nicole Edelman}

URL : http://journals.openedition.org/rh19/626

DOI : 10.4000/rh19.626

ISSN : $1777-5329$

\section{Éditeur}

La Société de 1848

\section{Édition imprimée}

Date de publication : 1 juin 2004

Pagination : 149-161

ISSN : 1265-1354

Référence électronique

Nicole Edelman, « Spiritisme et politique », Revue d'histoire du XIXe siècle [En ligne], 28 | 2004, mis en ligne le 07 avril 2008, consulté le 03 mai 2019. URL : http://journals.openedition.org/rh19/626 ; DOI : $10.4000 /$ rh19.626

Ce document a été généré automatiquement le 3 mai 2019.

Tous droits réservés 


\title{
Spiritisme et politique
}

\author{
Nicole Edelman
}

1 Une religion ou une croyance peut-elle susciter une réflexion ou une action politique? Dans le cadre d'un prosélytisme religieux, d'une défense de sa foi, les exemples ne manquent pas. Guerres saintes ou croisades, prises de pouvoir légitimées par Dieu, débats, polémiques, mises en accusation, voire mise à mort... L'ouverture vers une pensée critique est plus problématique mais elle accompagne bien souvent la naissance de religions. L'écriture des Évangiles, la protestation de Luther et plus près de notre siècle, l'apparition du spiritisme en sont des exemples probants.

En 1857, Hippolyte Léon Denizard Rivail, plus connu sous son pseudonyme d'Allan Kardec

${ }^{1}$ (1804-1869) publie Le Livre des Esprits. Cet ouvrage marque la naissance d'une nouvelle croyance: le spiritisme. Allan Kardec se dit le fondateur d'un nouveau christianisme, adapté à la modernité du XIX ${ }^{e}$ siècle : après Moïse et Jésus, des entités spirituelles s'adressent aux hommes pour leur apporter une troisième Révélation. Pour faire entendre ce message, ces esprits de l'au-delà choisissent des médiums, très généralement des femmes ${ }^{2}$ qui se mettent à leur écoute et transcrivent leurs discours selon différentes méthodes. Aux origines : des coups frappés dans les murs ou les planchers ${ }^{3}$ puis par les pieds de tables. Les "tables tournantes" se soulèvent et frappent des coups qui sont décodés à l'aide de l'alphabet. Rapidement, ce moyen, lent et fauteur d'erreurs, cède la place à une planchette légère montée sur roulette qui se meut sous la main du médium ${ }^{4}$. Enfin, l'écriture automatique (ou «mécanique ») permet de transcrire directement les pensées venues de l'au-delà, l'esprit s'emparant de la main du médium. Cette nouvelle technique implique un état modifié de conscience, qu'on pourrait qualifier d'autohypnose.

Les premières médiums spirites sont ainsi pour la plupart d'anciennes «somnambules magnétiques». Il existe en effet une continuité certaine entre les unes et les autres. Le somnambulisme magnétique (on l'appellera «hypnose » une soixantaine d'années plus tard) a été découvert en mai 1784 par Armand Marc-Jacques de Chastenet, marquis de Puységur. Cet aristocrate soignait par le magnétisme animal dont il avait appris les secrets avec le médecin viennois Franz Anton Mesmer. Pour ce dernier, l'univers baigne dans un fluide universel qui pénètre tous les corps animés, minéraux ou végétaux. Des 
« courants magnétiques » se propagent à travers l'espace et agissent sur l'ensemble des individus. Leur flux harmonieux au sein des corps est la condition nécessaire d'une bonne santé. Le médecin magnétiseur a donc pour tâche de maintenir ou de rétablir cette circulation grâce à des "passes » magnétiques qui provoquent bien souvent des crises convulsives et salvatrices. Or, lorsque le marquis de Puységur magnétise l'un de ses valets de ferme malade, loin de le faire convulser, il le plonge dans un étrange sommeil où il parle. Dans cet état modifié de conscience, le jeune garçon converse avec le marquis, diagnostique sa maladie et prescrit les soins qui doivent lui être donnés. Il annonce (avec justesse) la date de sa guérison. Puységur nomme ce nouvel état: "somnambulisme magnétique ».

4 Ces somnambules magnétiques sont à la fois visionnaires, voyants et guérisseurs ${ }^{5}$. Comme les médiums, ils sont majoritairement composés de femmes ${ }^{6}$. Depuis $1 \mathrm{a}$ fin du $\mathrm{XVIII}^{\mathrm{e}}$ siècle, ces somnambules disent converser avec des esprits extra-terrestres. Elles transcrivent (certaines écrivent déjà en « écriture automatique " ${ }^{7}$ ) ou font transcrire par celui qui les magnétise les textes qu'elles entendent. Les médiums spirites poursuivent donc les mêmes pratiques. Elles sont sans doute plus admirées que leurs sœurs somnambules puisqu'elles sont essentielles aux cercles spirites, ce qui leur confère un rôle et une place pastorale. Protégées par leur état modifié de conscience, elles discourent et sont écoutées. Elles écrivent et leurs écrits sont publiés.

5 Les médiums spirites sont ainsi en grande partie à l'origine des textes spirites majeurs, en particulier ceux de tous les grands théoriciens : d'Allan Kardec à Léon Denis, en passant par Gabriel Delanne. Aucun de ces hommes n'est en effet médium. Chacun a donc besoin de ces intermédiaires pour communiquer avec les esprits de l'au-delà. De même La Revue spirite est largement écrite par le canal de ces femmes.

6 Les premiers écrits touchent à la "religion" spirite dont les médiums élaborent la doctrine sous le feu des questions d'Allan Kardec qui, bien sûr, oriente par là même les réponses. Le spiritisme se veut donc une troisième Révélation, absolument moderne par sa nature que Kardec donne comme scientifique. Kardec affirme en effet procéder dans son élaboration comme les sciences expérimentales: les tables "parlent " parce qu'elles sont mues par des esprits intelligents. Toute autre explication est pour lui irrationnelle. Le spiritisme a ainsi pour ambition de renouveler le christianisme par la réconciliation de la science et de la religion. Le dogme catholique est alors revisité. Ancien et Nouveau Testament sont relus, commentés et interprétés. Purgatoire, paradis et enfer ne sont plus que des états relatifs. Le péché originel comme la nature divine du Christ sont récusés. La voix des esprits via les médiums réécrit l'histoire de l'humanité. Les hommes ne sont sur terre que de passage : ils viennent d'ailleurs et iront vers d'autres mondes. Le spiritisme pose en effet la réincarnation comme postulat fondamental. De vie en vie, les êtres humains progressent, chacun à son rythme, ne régressant jamais, au pire demeurant au même stade. Ils vont ainsi de lieux en lieux, selon une hiérarchie des planètes très précise, vers des espaces toujours plus immatériels, expiant peu à peu leurs fautes. Désincarnés, purs esprits, leur parcours se termine auprès du divin. Nullement inactifs, ces esprits supérieurs ont pour tâche d'aider ceux qui ne sont pas encore parvenus à ce stade ultime. Envoyés en mission charitable ou d'éducation, leur "périsprit», sorte d'enveloppe matérielle de l'esprit, peut les rendra visibles aux habitants de la terre s'il en est besoin. Ainsi vont les fantômes...

7 Dans un premier temps, celui du fondateur Allan Kardec, presque celui du Second Empire, les sociétés spirites se créent en milieu urbain, en particulier à Paris et Lyon. Elles 
promeuvent une solidarité mutuelle en soutenant l'ouverture de crèches, de caisses de secours, de bibliothèques, de dispensaires, de maisons de retraites. Elles se développent ainsi dans un milieu populaire. La voix des femmes est perceptible dans des revendications concernant le mariage, la famille et les enfants. Le Livre des Esprits ou plus souvent $L a$ Revue spirite mettent ainsi en question l'indissolubilité du mariage. Ces femmes spirites relativisent la filiation: un bébé n'est que le fruit de l'association d'un esprit qui vient d'ailleurs incarné dans un corps de chair au moment précis de la naissance. L'avortement perd ainsi tout caractère de gravité puisque le corps du fotus n'est qu'une enveloppe de chair vide ${ }^{8}$.

8 Par ailleurs, l'idée de réincarnation, qui peut s'effectuer dans le corps d'un homme ou d'une femme, d'un Noir ou d'un Blanc, oblige les croyants spirites à réfléchir à ce que signifie l'égalité. Les questions posées sur ce thème sont nombreuses, par les femmes mais aussi par les hommes conscients des risques d'un possible changement de sexe dans une vie future. Tous prônent l'importance de l'éducation, condition indispensable à l'émancipation. Beaucoup s'appuient sur le rôle maternel d'éducation des femmes pour la promouvoir. Tous rappellent l'égalité des droits entre les sexes mais la plupart ne transgresse pas l'idée d'une complémentarité entre homme et femme. Ainsi, en juin 1867, La Revue spirite rend compte de l'obtention du droit de vote des femmes à l'âge de 21 ans dans l'État du Wisconsin aux États-Unis et leur accès à des emplois publics. Sollicité de donner son avis, Kardec rappelle qu'hommes et femmes n'ont pas les mêmes attributions dans la société d'ici-bas. La solidarité est en revanche indispensable. Si chacun a mérité sa place sur la terre en raison d'une vie antérieure, chacun doit chercher à progresser luimême en faisant progresser les autres.

9 Ce militantisme ne sépare donc pas le social du politique. Le spirite se définit en tant qu'individu, comme membre d'une famille et comme citoyen. «Sauf exception, on peut donc admettre comme règle générale que tous ceux qu'une tâche commune réunit dans une existence ont déjà vécu ensemble pour travailler au même résultat et se trouveront réunis dans l'avenir jusqu'à ce qu'ils aient atteint le but, c'est à dire expié le passé ou accompli la mission acceptée ${ }^{9}$.

10 Il n'est pas possible de parler d'engagement politique dans ce premier temps du spiritisme mais cette nouvelle croyance est un vecteur fort de prise de parole et de réflexion critique, en particulier pour les femmes médiums. Je comparerai à d'autres situations de type religieux. On sait ainsi le rôle de la religion nouvelle dans le succès des saint-simoniens dans les années 1830, auprès des femmes en particulier. Le christianisme semblait alors à bout de souffle à beaucoup ${ }^{10}$. Les saint-simoniens se proposent «de reconstruire ce monde défait. La doctrine nouvelle doit se répandre et la religion se diffuser partout où elle est attendue, partout où elle manque. [...] Comme le dit Jeanne Deroin, le saint simonisme n'est pas un simple système politique, ni une doctrine politique, c'est une religion, une religion d'amour $"{ }^{11}$.

11 D'une certaine façon, le spiritisme joue un rôle comparable à la fin des années 1850. Les premiers spirites et les premières médiums spirites en particulier, s'appuient sur cette nouvelle religion pour prendre la parole, une parole bien souvent critique. Elles ont pour la plupart, comme toutes les femmes, une familiarité avec le christianisme à laquelle s'ajoute pour toutes la pratique du somnambulisme magnétique. Or le magnétisme animal, de par ses condamnations officielles par les Académies de médecine et des sciences en 1784 et en 1837, est un mouvement de pensée dissident qui permet et autorise même une pensée alternative. Beaucoup de ces femmes somnambules, avant même que le 
spiritisme ne se constitue en religion, disent entrer en contact avec différents esprits qui leur communiquent un savoir. Ces esprits ont bien souvent pour nom Saint-Simon ou Fourier ce qui implique, me semble-t-il, une familiarité préalable qui s'est sans doute traduite par des lectures ou des conversations ${ }^{12}$. Enfin, l'état modifié de conscience dans lequel elles sont réellement (ou disent être) leur confère un masque protecteur propice à la prise de parole ou à l'écriture, par ailleurs difficiles d'accès aux femmes du XIX ${ }^{\mathrm{e}}$ siècle, on le sait.

12 Le spiritisme serait donc pour ces femmes un moyen, original et détourné certes, pour des prises de positions politiques et sociales. Le catholicisme exclut les femmes de toute responsabilité sacerdotale et de toute prise de parole publique. Le protestantisme a en revanche une position parfois différente. On sait le rôle des "Grands réveils », à la fin du XVIII ${ }^{e}$ siècle et dans les années 1820 aux États-Unis par exemple, dans la redéfinition des rapports sociaux au sein de la famille. Les idées évangéliques ouvraient alors un grand débat sur la place revenant aux femmes. Elles encourageaient à une participation à la vie publique, sociale et politique. Le second "Grand Réveil», en particulier, féminise fortement la religion. Les Shakers affirment même la dualité de la nature divine : Dieu est à la fois homme et femme. Rien d'équivalent en France si ce n'est justement le spiritisme, qui voit cependant le jour à un autre moment chronologique et dans une culture différente par bien des traits.

13 Après la mort de Kardec, le mouvement spirite perd sa cohérence. La guerre de 1870 et le mouvement communal, Communes de Paris et de Lyon, confrontent la doctrine spirite élaborée à une action politique et sociale sanglante. Les spirites interprètent alors la violence comme un facteur de rupture qui ouvre plus grande la voie aux esprits en permettant aux hommes d'entendre leurs messages et donc de progresser plus vite. Aucune parole de révolte, encore moins d'appel à la révolution, ne se fait jour. Bien au contraire beaucoup de femmes médiums privilégient les communications avec les morts. Elles se replient alors sur l'au-delà et ses promesses.

Certaines cependant, avec quelques hommes, maintiennent une réflexion et une pensée sociale et politique. À Paris, des groupes spirites demeurent actifs autour de PierreGaétan Leymarie, successeur de Kardec à la direction de La Revue spirite, républicain exilé au Brésil après le coup d'État de Louis-Napoléon Bonaparte puis revenu en France. À la fin de l'année 1871, La Revue spirite publie des communications médiumniques critiques à l'égard du système d'organisation sociale français. Certes, la Terre est un monde inférieur dans la hiérarchie de l'univers. L'inégalité sociale y est signe et condition de l'expiation mais l'excès de la misère empêche la progression des êtres incarnés. Une réforme sociale est donc nécessaire. Elle passe, pour Pierre-Gaétan Leymarie, par une plus large place donnée aux femmes dans la vie publique. Le directeur de La Revue spirite salue ainsi la reparution de L'Avenir des femmes, journal féministe modéré, réédité grâce à l'action de Léon Richer et de Maria Deraisme. Comme eux, Leymarie milite pour une réforme du Code civil afin que la femme trouve sa pleine et entière "personnalité morale et juridique ${ }^{13}$. Il souhaite une égalité de salaire entre hommes et femmes. En Algérie, où le spiritisme s'est bien implanté, une spirite condamne, en 1870, la polygamie, les mariages obligés et l'excès du nombre d'enfants des femmes algériennes qui meurent trop tôt épuisées par les maternités et le travail. Elle plaide pour leur libération par le spiritisme ${ }^{14}$

15 La médium Antoinette Bourdin participe largement à l'écriture de la revue avec laquelle elle partage l'admiration pour le spirite Jean-Baptiste Godin et son familistère. Les 
romans qu'elle publie en portent trace. En 1874, Les deux sœurs, roman historique ${ }^{15}$ met en scène des patrons (spirites) associant leurs ouvriers aux bénéfices de leurs entreprises. Le village entier, hommes et femmes, ouvriers et paysans, est sociétaire de l'association communale dont le comité de gestion est élu au suffrage universel (vrai!). Des écoles mixtes, une bibliothèque, un dispensaire, un entrepôt de marchandises vendues au prix d'achat sont créés. L'anticléricalisme n'est même plus nécessaire puisque le curé est devenu spirite! Antoinette Bourdin appelle donc à l'action, ici et maintenant. «Puisque nous sommes sur la terre, cherchons d'abord par tous les moyens possibles à nous rendre ce séjour agréable et utile en accomplissant le devoir du dévouement envers la société [... ]. À l'œuvre donc! que des hommes influents prennent l'initiative pour grouper autour d'un grand centre toutes nos bonnes volontés et utiliser les moyens qui sont à notre disposition ${ }^{16}$.

En 1890, le "Groupe spirite bisontin " s'intéresse encore à la situation des prolétaires certes responsables de leur incarnation dans cette malheureuse condition mais dont rien ne justifie le surcroît de misère infligé. Ces spirites craignent en effet la révolte de ces hommes (et peut-être craignent-ils l'influence grandissante de Marx en France). Or toute action violente conduit de facto à une régression dans une vie future. Aussi préconisent-ils de nombreuses réformes, qui ressemblent fort aux projets proudhoniens, en particulier la création ou le développement de coopératives agricoles et industrielles et la participation des ouvriers aux bénéfices des entreprises ${ }^{17}$. Ce "Groupe bisontin » exprime sans doute un des derniers échos de ce spiritisme socialiste, réformiste, fraternel et sororal. Pendant ces décennies 1870-1890, charité, dévouement, entraide sont donc requis, et ce, par delà les frontières.

Cette volonté de fraternité universelle constitue en effet aussi l'un des caractères du spiritisme de ces années. Ces spirites ne partagent ni l'esprit nationaliste et antiallemand, ni d'ailleurs l'antisémitisme, qui prennent alors tant d'ampleur.

Une nouvelle inflexion voit alors le jour à Tours où Léon Denis (1846-1927) forme un groupe spirite en 1893. Léon Denis aurait découvert Le Livre des Esprits à 18 ans, lecture qui l'aurait convaincu de la véracité du spiritisme. L'écoute d'une conférence de Kardec de passage dans la ville en 1867 aurait achevé sa conversion. Son charisme, ses qualités littéraires et oratoires font de lui un chef spirite largement reconnu. Sir Arthur Conan Doyle le décrit comme un "maître vénéré ", physiquement impressionnant: "d'une solide et forte carrure, [avec] un air majestueux, [une] tête léonine qui rappelait ces vieux prêtres celtiques ou ces guerriers primitifs ${ }^{18}$. Léon Denis est le premier depuis Allan Kardec à publier des ouvrages spirites théoriques. Comme Kardec, Denis n'est pas médium, il collabore essentiellement avec une femme médium, très douée, Mme Forget ( sic) dont il regrette la mort en 1917. La symbiose intellectuelle entre eux a du être particulièrement forte. Ce type de rapport n'a pas existé autour de Kardec qui a toujours eu plusieurs médiums, dont aucune attitrée, ce qui, sans doute, leur a laissé plus d'autonomie.

19 Ces communications mettent au jour un spiritisme qui accepte, et valorise même dans un premier temps, le régime nouveau républicain: son cadre libéral et anticlérical et ses capacités d'assurer l'ordre social, en particulier. Les velléités de réforme politique et sociale sont alors mises en sourdine. Puis, lorsque les radicaux arrivent au gouvernement, Léon Denis ne cesse de déplorer l'anarchie morale et sociale et l'incohérence des idées philosophiques de son temps, qu'elles soient prônées par l'Église ou l'Université, qu'il finit par mettre sur le même plan. Il fulmine contre le matérialisme, contre la décadence, 
contre «le relâchement du caractère et de l'énergie vitale de notre race ${ }^{19}$. " La démocratie, radicale ou socialiste, dans ses masses profondes et dans son esprit dirigeant, s'inspirant elle aussi, des doctrines négatives, ne pouvant aboutir qu'à un résultat négatif pour le bonheur et l'élévation de l'humanité. Tant vaut l'idéal, tant vaut l'homme, tant vaut la Nation, tant vaut le pays ${ }^{20}$. Or le socialisme, cible privilégiée de ses attaques, voulant imposer l'égalité, peut au pire aboutir à l'anarchie, au mieux être inefficace. « Parmi les systèmes préconisés par les socialistes pour amener une organisation pratique du travail et une sage répartition des biens matériels, les plus connus sont la coopération, l'association ouvrière; il en est même qui vont jusqu'au communisme. Jusqu'ici, l'application partielle de ces systèmes n'a produit chez nous que de maigres résultats [...]. La cause du mal est en nous, dans nos passions, dans nos erreurs. C'est là ce qu'il nous faut changer. Pour améliorer nos sociétés, il faut améliorer l'individu " ${ }^{21}$.

Comme les catholiques, il valorise le rôle de la souffrance et de la douleur. D'une part, souffrance et douleur étant largement partagées par les êtres humains, il suffit de regarder quelqu'un de plus malheureux que soi pour relativiser son malheur. D'autre part, le spiritisme permet de comprendre sa souffrance, de l'accepter sans chercher à y surseoir au risque de mettre en péril sa vie suivante. Enfin, la mort étant la vie, les deuils sont joyeux... "Le spiritisme nous montre dans les malheureux des apprentis, des néophytes que la douleur éclaire, initie, des candidats à la perfection et au bonheur " ${ }^{22}$.

21 Enfin, Léon Denis s'intéresse à la nation, une nation en décadence depuis peu, «à volonté faible et changeante [...]. Le pessimisme et le matérialisme, qui se répandent de plus en plus parmi nous, tendent encore à amoindrir les qualités généreuses de notre race. Le positivisme et l'agnosticisme travaillent systématiquement à éteindre ce qui restait de viril dans l'âme française [...] ${ }^{23}$. Cette question nationale se manifeste chez l'ensemble des spirites après la guerre franco-prussienne de 1870. Elle ne cesse ainsi de parcourir les « instructions » médiumniques mais avec des sensibilités politiques différentes. Quelques années avant la Grande guerre, la baronne de Watreville est ainsi bien plus positive que Léon Denis : «J'aime la France, parce qu'elle s'est faite ce qu'elle est, c'est à dire bonne, humaine, républicaine et égalitaire ${ }^{24}$.

Pour Denis, la France est une nation privilégiée qui a, dans la transmission du spiritisme au monde, un rôle particulier à jouer. « Par elle-même, la France est une et multiple, écrit en 1893 la médium Mme Hyver. Chaque province présente un type particulier d'activité humaine et toute la race se trouve néanmoins puissamment centralisée. [...] Le rôle de la France sera merveilleux, car elle étendra son pouvoir moral sur toutes les nations, du Nord au midi, de l'Est à l'Ouest; elle combattra pour la justice. C'est elle qui introduira l'idée religieuse dans la vie sociale ${ }^{25}$.

Le spirite Léon Denis et les médiums qui l'entourent transforment ainsi l'idée fraternelle et cosmopolite originelle du système kardéciste. Ils valorisent l'idée de patrie au titre de l'apport de chaque pays à l'unification religieuse de l'humanité. Un message médiumnique de Jeanne d'Arc, que Léon Denis vénère, souligne même l'importance de la concurrence et de l'émulation entre les nations : « qui les stimule et les féconde, les élève à leur suprême puissance ${ }^{26}$. Aussi Léon Denis, vingt ans après la guerre francoprussienne, ne craint-il pas un nouveau conflit armé. « Tout en déplorant les maux causés par la guerre, nous ne tombons pas pour autant dans un "pacifisme » débilitant. Pour assurer l'intégrité morale et matérielle de la France, nous reconnaissons encore la nécessité d'une armée, que les progrès de la civilisation permettront peut-être d'employer un jour à des œuvres d'utilité générale ${ }^{27}$. En effet, selon Jeanne d'Arc, au 
final, «se dégagera un jour après une lente, confuse et douloureuse incubation, l'âme des grandes patries. De leur réunion naîtra une civilisation, dont celle des temps présents n'est que l'ébauche grossière ${ }^{28}$.

Ces grandes patries sont composées de l'Inde, de l'Égypte, de la Grèce et de la Gaule. Le peuple d'Israël s'y distingue et joue un rôle-clé : « Moïse, l'initié d'Égypte, guidait vers la Terre promise le peuple par qui la pensée monothéiste allait entrer dans le grand mouvement religieux et se répandre sur le monde. Le rôle du peuple d'Israël est considérable ${ }^{29}$. D'autant plus que les Juifs ont compris que Jésus n'était pas Dieu. Le christianisme a ainsi dévoyé la parole du Christ pourtant essentielle apportant les idées d'humanité et d'éternité. Léon Denis déplore donc le rôle du catholicisme et son interprétation erronée de la révélation de Jésus. Il critique enfin le positivisme en ce qu'il a cru pouvoir fonder une morale détachée de toute religion. Le spiritisme, en revanche, s'attache à nouer philosophie, science et religion. « Incomparable instrument d'analyse, il n'invente rien, il constate. En tant que science expérimentale, le spiritisme est soumis à la loi du progrès ${ }^{30}$. Il permet de faire la synthèse de toutes les grandes religions précédentes dans cet esprit de progrès qui trouve enfin la plénitude de son sens. Le spiritisme devrait donc être la religion de la République, conciliant science et morale.

Le discours de Léon Denis construit ainsi une morale qui exclut toute action politique et sociale. Denis défend une philosophie fortement individualiste au nom même du spiritisme dont la loi fondamentale est, en effet, celle de l'évolution individuelle et progressive. Certes, il ne gomme pas totalement la fraternité prônée par les premiers spirites mais il ne propose plus qu'une union des cœurs et non une action immédiate de transformation. Il est de toute façon convaincu que la certitude de renaitre de multiples fois dans des conditions différentes obligera bientôt chacun aux compromis. En effet, si le spiritisme était vainqueur, il n'y aurait plus de question sociale. «Les concessions entre classes deviendraient faciles, et l'on verrait cesser tout antagonisme entre le capital et le travail. La vérité étant connue, on comprendrait que les intérêt des uns sont les intérêts de tous et que nul ne doit être la proie des autres " ${ }^{31}$. Plus de haine mais justice, confiance, estime et affection. Léon Denis estime par ailleurs que la «question sociale n'embrasse pas seulement les rapports des classes entre elles, elle concerne aussi la femme de tous les rangs, la femme cette grande sacrifiée, à laquelle il serait équitable de rendre ses droits naturels, une situation digne d'elle, si on veut voir la famille plus forte, plus morale, plus unie ${ }^{32}$. Rien de plus cependant : ni émancipation, ni indépendance.

Ce spiritisme tourangeau n'a donc plus rien de dissonant, ses conceptions de la femme font écho à la pensée dominante de ce début du XXe siècle. Léon Denis modifie ainsi le dogme de la réincarnation proposé par les premières médiums kardécistes. Ses propres " guides" spirituels lui ont en effet appris (tardivement) qu'il est préférable de toujours se réincarner dans le même sexe, que le contraire est inutile et même dangereux. Il est d'ailleurs « facile, écrit-il, de reconnaître à première vue, autour de nous, les personnes qui, dans une existence précédente, avaient adopté un sexe différent; ce sont toujours, à quelque point de vue, des anormaux ${ }^{33}$.

Léon Denis enferme le spiritisme dans un cadre religieux: l'espoir de vies futures meilleures sous-tend la vie présente. À chacun de faire connaître cette nouvelle religion. Lui-même s'y emploie en multipliant les ouvrages et les conférences.

À Paris ou à Lyon, les groupes demeurent mieux insérés dans des préoccupations sociales du temps présent. Les voix des médiums ne sont pas monocordes à la différence de celle de Léon Denis. 
Rufina Noeggerath (1821-1908) s'intéresse ainsi d'abord aux preuves de survie après la mort. Son souci n'est pas d'ordre religieux : il se veut scientifique. «La survie est une vérité prouvée, indéniable, en dehors de tout dogme ${ }^{34}$. Elle connait bien le magnétisme animal et le somnambulisme magnétique sans doute parce que son époux était médecin et magnétiseur. Veuve depuis 1852, elle a très vite cherché à communiquer avec son défunt mari, ce qui l'a conduit assez naturellement au spiritisme à la fin du Second Empire. Médium douée, elle crée alors son propre groupe spirite. Ses communications sont fortement marquées par la tolérance et l'anti-cléricalisme. Elle y défend tous les persécutés, les Juifs en particulier. Dans son livre La survie, sa réalité, sa manifestation, sa philosophie, publié en 1897, elle ne cite pas Dreyfus (mais l'Affaire ne commence vraiment qu'en 1898). Ses plaidoyers sont en revanche très argumentés: elle souligne, comme Denis, que les Juifs ont su reconnaître que le Christ n'était pas Dieu. Les spirites pensent en effet qu'il n'est qu'un grand homme, réincarnation d'un esprit supérieur envoyé en mission sur terre pour faire évoluer plus vite ses habitants. Aussi anathémise-t-elle l'Église qui «a accablé les Juifs de ses malédictions, [qui] les a outrageusement persécutés; les rois ont aidé l'église à les spolier, mais malgré les massacres et les persécutions, les Israélites se sont répandus dans le monde et leur nombre ne fait qu'augmenter. [...] Dispersés au milieu de toutes les nations, les Juifs vivent de par la loi des harmonies. Il faut qu'ils restent dans le monde; ils y resteront. Savez-vous pour quelle raison puissante ? C'est parce que les Juifs sont la preuve vivante que Jésus est venu sur terre et qu'il n'était point Dieu. [...] Depuis bientôt dix-neuf siècles, les Juifs sont une protestation perpétuelle contre le blasphème des chrétiens qui ont fait d'un homme... un Dieu ! ! ! ${ }^{35}$ Il est donc probable que Rufina Nœggerath a soutenu Dreyfus, mais je n'en ai pas retrouvé trace. La seule prise de position écrite est celle des Lyonnais. En 1899, dans la revue La paix universelle, Jean Bouvery, Daniel Metzger et Paul Grendel appellent tous les spiritualistes à soutenir Dreyfus dans sa lutte.

La croyance spirite en la réincarnation et la pluralité des mondes devrait logiquement promouvoir la tolérance. Le fonctionnement des spirites en petites sociétés, fondées sur l'interrogation des esprits censés venir améliorer la vie des hommes, aiguise sans doute la curiosité intellectuelle des membres tant dans le questionnement que dans la compréhension des réponses. Les interrogations, lorsqu'elles ne portent pas sur des questions intimes, relèvent de préoccupations religieuses, sociales ou politiques. Elles reflètent probablement les problèmes du quotidien de ces hommes et de ces femmes. Ces dernières, souvent médiums, sont donc incitées à porter un intérêt particulier au monde extérieur, à sortir du cadre familial qui traditionnellement est le leur. Elles doivent en effet faire face à des questions parfois complexes. Certes, elles y répondent sous la protection de leur médiumnité qui les aide à parler, mais encore faut-il qu'elles aient quelque chose à dire ! Or, elles savent qu'elles seront jugées sur le discours prononcé. Plus le siècle avance, plus elles sont immergées dans des informations complexes. Les revues, La Revue spirite en particulier, traitent de problèmes très divers. Les recherches scientifiques, psychiques par exemple, y tiennent une place de plus en plus en grande au fil des découvertes neurologiques et psychologiques des médecins. La lecture de ces rubriques nécessite donc des connaissances qui dépassent le cadre habituel de l'horizon féminin (et bien souvent masculin). Au début du XXe siècle, certain-e-s débattent ainsi de sujets aussi différents que l'existence de l'inconscient, de la Triplice ou encore de l'alliance franco-russe et de la guerre russo-japonaise ${ }^{36}$. 
31 La question du suffrage " universel » est récurrente des années 1890 à la Première guerre mondiale. Elle est à la fois posée par des hommes et par des femmes. La Revue spirite se fait l'écho de réflexion et de discussions sur ce thème. Elle reflète assez précisément les questionnements des féministes françaises mais aussi anglo-saxonnes. Les médiums (de deux sexes) débattent de l'âge requis qui permettrait aux femmes de voter. Devraientelles être électrices et éligibles ou seulement électrices? Le seraient-elles à tous les échelons de la vie publique ou seulement au niveau municipal? Devraient-elles être mariées? Mères de surcroît? Célibataires? En 1896, Pierre-Gaétan Leymarie envisage ainsi une représentation politique des femmes au sein d'un collège électoral spécial, sur le modèle des projets de Jean-Baptiste Godin dans son familistère de Guise. À ce " sénat » féminin se joindrait une assemblée d'hommes. La société serait alors représentée dans sa plénitude et le terme de "suffrage universel » ne serait plus un mensonge. "Le suffrage politique, dit universel, ne peut l'être que si la femme est admise à voter ", écrit Leymarie en $1896^{37}$. Claire Galichon, médium et écrivain, publie un certain nombre d'ouvrages où elle défend un "féminisme spiritualiste ». En 1909, en matière de suffrage, elle dénonce violemment le suffrage « unisexuel ». « En face du concours général des avis, des vœux et des volontés, la voix de la femme n'a pas le droit de s'élever. Fût-elle un génie, elle doit s'effacer devant le crétin; à la plus remarquable des femmes est refusé le droit que possède le plus ignare, le plus incapable des hommes " ${ }^{38}$. Elle souhaite un "suffrage universel proportionnel » égal entre homme et femme de plus de 21 ans (elle évoque même la possibilité d'âges plus avancés), en rapport avec leur valeur personnelle. Seul-e-s voteraient ceux et celles qui en auraient les capacités morales et les compétences intellectuelles ${ }^{39}$. Aussi dans un premier temps, Claire Galichon demande le droit de vote pour les femmes dans ces conditions puisque toutes les femmes ne sont pas aptes à voter (certes pas plus que les hommes). Elle argumente au nom du cœur et du sentiment, de la maternité mais aussi de l'intelligence des femmes (et des hommes). Elle plaide en effet pour une représentation " par la cime, c'est-à-dire par des intelligences qui ont fait leurs preuves. Ce serait en quelque sorte, la synarchie asexuelle, mise à la place de la démocratie uni-sexuelle, autrement dit, la société dominée par le savoir, l'expérience et la raison des deux sexes, au lieu d'être dominée par l'ignorance et l'appétit individuels des foules masculines ${ }^{40}$.

32 En 1910, Pulvis, dit Algol, un homme, est, lui aussi, radical dans ses propos. Il use des mots même des premières féministes saint-simoniennes en 1848 : «Singulier universalisme que celui qui prive de ce droit toute une moitié du genre humain, tout un sexe à qui l'autre doit la vie! Mais avant la Révolution, ils n'avaient pas ce droit. Comment ne se sont-ils pas aperçus qu'ils s'étaient octroyés un monstrueux et abusif privilège ${ }^{41}$. Si ces hommes sont particulièrement exceptionnels, le lien entre féminisme, politique et spiritisme l'est moins. La journaliste Olympe Audouard, Maria Pognon, Claire Galichon et plus tard Hélène Brion ont cette double appartenance. Le spiritisme autoriserait donc et favoriserait même une réflexion autonome, et sur le politique et sur la religion, sur le sens des mots aussi. Bien des femmes spirites se permettent ainsi des pensées critiques par le biais de leur médiumnité. Claire Galichon l'explique dans un de ses ouvrages. « Nos religions dogmatiques avec leur enseignement caducs, leur morale fausse, nos philosophies positives ou abstraites auraient-elles pu me faire trouver la solution possible de tant d'incohérences sociales qui victimisent la femme? Je ne le crois pas. [...] Le spiritualisme est forcément révolutionnaire (parce qu'il affirme que toutes les créatures humaines sont des émanations de Dieu et donc toutes les mêmes droits). Ne faut-il pas 
qu'il attaque les dogmes divers ceux de la théologie comme ceux du matérialisme qui ont accumulé mensonges sur mensonges $[. ..]{ }^{42}$. Le tremplin de ce qu'elle appelle un « spiritualisme indépendant » l'autorise à formuler des critiques de tous ordres. Elle est d'ailleurs persona non grata à La Revue spirite qui la juge sans doute excessive dans ses propos. Elle tient une rubrique féministe dans la revue L'Étoile (qui devient L'Âme) de René Caillée, le fils homonyme de celui qui se rendit à Tombouctou; peu de temps puisque René Caillée meurt après qu'il lui a ouvert les colonnes de sa revue. Elle n'en poursuit pas moins son travail qu'elle publiera sous forme de livre. Elle y critique systématiquement les thèses qui infériorisent physiquement, intellectuellement et psychiquement la femme, citant divers travaux de médecins et d'anthropologues. Elle réclame une éducation et une instruction égales pour les deux sexes, un salaire égal lorsque le travail est égal, l'accès des femmes à toutes les carrières. Elle réclame enfin une forte modification du Code civil qu'elle connaît fort bien et surtout des lois qui régissent le mariage. Elle dénonce l'assujettissement de l'épouse et la violence du mari qui commence bien souvent lors de la nuit de noce. "Certaines nuits de noce sont des nuits de bataille. [...] Ce qui importe en face du désir d'amour, c'est qu'il soit réciproque. Quand il est partagé, il perd tout caractère répugnant ${ }^{43}$. Elle met ainsi en accusation la morale, une double morale, hypocrite et «masculiniste », terme qu'elle invente pour désigner ceux qui combattent la femme.

La croyance d'être un esprit incarné transitoirement dans un corps de chair, la pensée d'une possibilité de changer de sexe de vies en vies, celle de communiquer directement à des esprits supérieurs, véritables "guides » spirituels, modifient donc très probablement un mode d'être et de penser. C'est, du moins, l'hypothèse que je fais ici. Ces croyances et ces conceptions, souvent fortement dissonantes par rapport à la pensée dominante, permettent peut-être de ne pas intérioriser une infériorisation et d'acquérir la certitude d'être capable de penser par soi-même, ce qui ne va pas de soi.

\section{NOTES}

1.. Selon les dires d'une de ses médiums, il aurait porté ce nom en tant que druide celte dans une vie antérieure.

2.. Je féminiserai le mot puisque les médiums sont majoritairement des femmes.

3.. Les sœurs Fox entendent ces coups frappés (raps) à Hydesville aux États-Unis au printemps 1848. Avec leur mère, elles en transcrivent le sens par une méthode alphabétique.

4.. Planchette appelée aussi le « oui-Ja ».

5.. Voir Nicole EDELMAN, Voyantes, guérisseuses, et visionnaires en France, 1785-1914, Paris, Éditions Albin Michel, 1995, 280 p.

6.. Je féminiserai donc aussi leur nom.

7.. Voir Christine BERGÉ, L'au-delà des Lyonnais. Mages, médiums et francs-maçons du XVIII au XXe siècles, Lyon, Éditions Lugd, 1995 et « Identification d'une femme : les écritures de l'agent inconnu » dans L'Homme, n 44, octobre-décembre 1997, pp. 106-129. 
8.. La Revue spirite, mars 1858.

9.. Allan KARDEC, Euvres posthumes, Paris, Éditions Dervy, 1978, p. 128. Il s'agit d'une réponse médiumnique.

10.. Voir Michèle RIOT-SARCEY, De la liberté des femmes. «Lettres de dames » au Globe, 1831-1832, textes recueillis et présentés par Michèle Riot-Sarcey, Paris, Éditions Côtéfemmes, 1992, $148 \mathrm{p}$.

11.. Ibidem, pp. 11 et 17.

12.. Voir Nicole EDELMAN, Voyantes, guérisseuses, et visionnaires, ouv. cité.

13. La Revue spirite, 1871, p. 654.

14.. H. V., La femme et la philosophie spirite, influence des croyances philosophiques sur la situation de la femme dans l'antiquité, le Moyen Âge et de nos jours, Paris, Librairie spirite, 1870.

15.. Antoinette BOURDIN, Les deux sœurs, roman historique, Genève, Éditions J. Benoit et Cie, 1874.

16.. Antoinette BOURDIN, Les souvenirs de la folie, Genève, Éditions J. Benoit, 1875, pp. 284 et 288 .

17.. La Revue spirite, juillet-septembre 1890.

18. Claire BAUMARD, Léon Denis intime, préface de Sir Arthur Conan Doyle, Paris, Éditions Jean Meyer, 1929, préface.

19.. Léon DENIS, Le problème de l'être et de la destinée, Paris, Éditions Leymarie, 1985 (1 ${ }^{\text {ère }}$ édition 1908), p. 4.

20.. Ibidem, p. 7.

21.. Léon DENIS, Après la mort. Exposé de la doctrine des esprits. Solution scientifique et rationnelle des problèmes de la vie et de la mort. Nature et destinée de l'être humain. Les

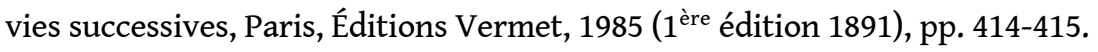

22.. Léon DENIS, Le problème de l'être et de la destinée, ouv. cité, p. 525.

23.. Ibidem, p. 427.

24.. Extrait de communications médianimiques, Paris, Éditions rue Gerbert, [1909], tome 3, p. 329. Les médiums sont des proches de Gabriel Delanne.

25.. Léon DENIS, Le Monde invisible et la guerre, Paris, Librairie des sciences psychiques, 1919, p. 247-248.

26. Léon DENIS, Jeanne d'Arc médium, Paris, Librairie des sciences psychiques, 1910, p. 224.

27.. Léon DENIS, Après la mort..., ouv. cité, p. 415, note 1.

28. Léon DENIS, Jeanne d'Arc médium, ouv. cité, p. 225.

29.. Léon DENIS, Après la mort, ouv. cité, p. 73.

30.. Deuxième Congrès spirite universel, compte-rendu sténographique organisé par le Bureau international du spiritisme, Genève, Jent, 1913, p. 33.

31. Ibidem, p. 417.

32. Ibidem, p. 420.

33. Ibidem, p. 232.

34.. Rufina NOEGGERATH, La survie, sa réalité, sa manifestation, sa philosophie. Échos de l'audelà, préface de Camille Flammarion, Paris, Librairie des sciences psychiques, 1897, p. XI. 35. Ibidem, p. 328. La communication est signée... l'abbé Grégoire. Rufina Noeggerath est manifestement une femme très cultivée. Une communication retraçant la vie d'une sorcière rappelle ainsi trait pour trait le plan général de La Sorcière de Michelet.

36.. Il s'agit de la baronne de Watreville et deux de ses consœurs médiums dans Extrait de communications médianimiques, ouv. cité, tome 2, pp. 317 et 330.

37.. La Revue spirite, 1896, p. 653. 
38.. Claire GALICHON, Ève réhabilitée, plaidoyer " pro femina », Paris, Librairie des sciences occultes, 1905, p. 158.

39.. Ibidem, p. 160.

40.. Ibidem, p. 172. Elle place en note les pays où les femmes ont déjà le droit de vote à cette date, notant en particulier les différents États des États-Unis.

41.. La Revue spirite, 1910, p. 365.

42.. Claire GALICHON, Ève réhabilitée..., ouv. cité, pp. 17 et 88.

43.. Ibidem, p. 399 et p 405.

\section{RÉSUMÉS}

Une religion ou une croyance peut-elle susciter une réflexion ou une action politique? Dans le cadre d'un prosélytisme religieux, d'une défense de sa foi, les exemples ne manquent pas: guerres saintes ou croisades, prises de pouvoir légitimées par Dieu, débats, polémiques, mises en accusation, voire mise à mort. L'ouverture vers une pensée critique est plus problématique mais elle accompagne bien souvent la naissance de religions. L'écriture des Évangiles, la protestation de Luther et plus près de notre siècle, l'apparition du spiritisme en sont des exemples probants. L'article analyse les écrits du fondateur de cette religion, Hippolyte Léon Denizard Rivail, plus connu sous son pseudonyme d'Allan Kardec (1804-1869), puis de certains autres théoriciens, tel Léon Denis (1846-1927), ainsi que d'un certain nombre de femmes, Antoinette Bourdin ou Claire Galichon par exemple, dans leur rapport avec le politique.

Spiritualism and politics. Can a religion or a belief bring about political reflection or action? There are many examples in a context of religious proselytism or defence of the faith: holy wars or crusades, accession to power legitimised by God, debates, controversies, indictments, and even murder. The emergence of critical thought is more problematic, but it often goes along the birth of religions. The writing of Gospels, Luther's protest and, closer to our own century, the birth of spiritualism, are convincing examples of this emergence. This paper analyses, in their relations to politics, the writings of the founder of this religion, Hippolyte Léon Denizard Rivail, betterknown under his pen name, Allan Kardec (1804-1869), as well as the writings of a number of theoreticians of spiritualism, such as Léon Denis (1846-1927), as well as those of several women, including Antoinette Bourdin and Claire Galichon.

\section{AUTEUR}

\section{NICOLE EDELMAN}

maître de conférences à l'Université Paris 10 